\title{
AN OSCILLATION CRITERION FOR LINEAR SECOND-ORDER DIFFERENTIAL SYSTEMS
}

\author{
F.V. ATKINSON, HANS G. KAPER AND MAN KAM KWONG ${ }^{1}$
}

\begin{abstract}
This article is concerned with the oscillatory behavior at infinity of the solution $y:[a, \infty) \rightarrow \mathbf{R}^{n}$ of a system of $n$ second-order differential equations, $y^{\prime \prime}(t)+Q(t) y(t)=0, t \in[a, \infty) ; Q$ is a continuous matrix-valued function on $[a, \infty)$ whose values are real symmetric matrices of order $n$.

It is shown that the solution is oscillatory at infinity if (at least) $n-1$ eigenvalues of the matrix $\int_{a}^{t} Q(t) d t$ tend to infinity as $t \rightarrow \infty$.
\end{abstract}

1. Introduction. We are concerned with the differential equation

$$
y^{\prime \prime}(t)+Q(t) y(t)=0, \quad t \in[a, \infty),
$$

for a vector-valued function $y:[a, \infty) \rightarrow \mathbf{R}^{n}$. Here $Q$ is a continuous matrix-valued function on $[a, \infty)$ whose values are real symmetric matrices of order $n$.

Two points $\alpha, \beta \in[a, \infty)$ are said to be conjugate relative to (1.1) if there exists a nontrivial function $y$ which satisfies (1.1) and vanishes at $\alpha$ and $\beta$. (1.1) is said to be oscillatory at infinity if, for any point $\alpha \in[a, \infty)$, there exists a point $\beta \in(\alpha, \infty)$ such that $\alpha$ and $\beta$ are conjugate relative to (1.1). We use the notation $Q_{1}(t)$ for the matrix of the integrals over [ $a, t]$ of the corresponding elements of $Q(t)$,

$$
Q_{1}(t)=\int_{a}^{t} Q(s) d s .
$$

The oscillation theory for (1.1) has received considerable attention in the past; see, for example, Reid [7, Chapter V]. It has been conjectured that (see Hinton and Lewis [1]) (1.1) is oscillatory at infinity whenever

$$
\lim _{t \rightarrow \infty} \lambda_{1}\left\{Q_{1}(t)\right\}=\infty
$$

Here, $\lambda_{1}\{\cdot\}$ denotes the largest eigenvalue of the matrix inside the braces. At this point, the conjecture has been established for the case $n=2$ by Kwong and Kaper [2]. For arbitrary $n$, the conjecture has been established under various growth conditions on the trace of $Q_{1}(t)$ by Mingarelli [5, 6] and Kwong et al. [3].

Received by the editors August 16, 1983 and, in revised form, May 9, 1984.

1980 Mathematics Subject Classification. Primary 34A30, 34C10.

Kev words and phrases. Matrix differential equation, oscillation theory, matrix Riccati equation, Riccati inequality.

${ }^{1}$ This work was supported by the Applied Mathematical Sciences Research subprogram (KC-04-02) of the Office of Energy Research of the U. S. Department of Energy under Contract W-31-109-ENG-38. 
We shall study the oscillatory behavior of (1.1) by means of the Riccati equation for the matrix-valued function $R$,

$$
R(t)=R(a)+Q_{1}(t)+\int_{a}^{t} R^{2}(s) d s, \quad t \in[a, \infty) .
$$

A necessary and sufficient condition for (1.1) to be nonoscillatory at infinity is that (1.4) has a continuous solution $R$ on a half-line (which, without loss of generality, may be taken as $[a, \infty)$ ) whose values are selfadjoint matrices of order $n$; see $[2,3]$.

In this paper we prove that $(1.1)$ is oscillatory at infinity if (at least) $n-1$ eigenvalues of $Q_{1}(t)$ tend to infinity as $t \rightarrow \infty$,

$$
\lim _{t \rightarrow \infty} \lambda_{i}\left\{Q_{1}(t)\right\}=\infty, \quad i=1, \ldots, n-1 .
$$

Here each eigenvalue is counted according to its multiplicity. The proof is given in $\S 3 ; \S 2$ contains some preliminary material.

2. Preliminaries. Our main goal in this section is to establish an ordering relation for the quadratic term in the matrix Riccati equation

$$
R(t)=F(t)+\int_{0}^{t} R^{2}(s) d s, \quad t \geqslant 0 .
$$

Here $F$ is a given function defined on $(0, \infty)$, whose values are symmetric matrices of order $n$. We shall use the standard partial ordering in the space of selfadjoint matrices, viz., $A \geqslant B$ if $A-B$ is nonnegative. The symbol $I$ stands for the identity matrix of order $n$.

At several points in the discussion we shall need the following result from matrix theory.

LEMMA 1. If $A$ is a real symmetric matrix of order $n$ with eigenvalues $\mu_{1} \geqslant \mu_{2} \geqslant \cdots$ $\geqslant \mu_{n}, B$ is a nonnegative matrix of order $n$, and $A+B$ has eigenvalues $\lambda_{1} \geqslant \lambda_{2} \geqslant \cdots$ $\geqslant \lambda_{n}$, then $\lambda_{i} \geqslant \mu_{i}$ for $i=1, \ldots, n$.

Proof. The lemma is a consequence of the Courant-Fischer theorem; see [4, Theorem 3.6.3].

The next lemma contains an auxiliary result that we shall need in the proof of Lemma 3.

LEMMA 2. Let $A=\left(a_{i j}\right)$ be a real symmetric matrix of order $n \geqslant 2$ such that $A \geqslant 0$. If for some $k \in\left[2^{-1 / 2}, 1\right)$ the matrix $B=\left(b_{i j}\right)$, with elements

$$
\begin{array}{ll}
b_{i j}=k\left(a_{i j}+1\right) & \text { if } i=j, \\
b_{i j}=a_{i j} & \text { if } i \neq j,
\end{array}
$$

has $n-1$ eigenvalues $\leqslant 0$ (each eigenvalue counted according to its multiplicity), then $a_{i i}>1$ for $i=1, \ldots, n$.

Proof. Consider first the case $n=2$. Because $\operatorname{tr} B=k(2+\operatorname{tr} A)$ and $A \geqslant 0$, we have $\operatorname{tr} B>0$, so at least one eigenvalue of $B$ must be positive. Hence, since the other eigenvalue is negative or zero, det $B \leqslant 0$, i.e., $k^{2}\left(a_{11}+1\right)\left(a_{22}+1\right) \leqslant a_{12}^{2}$. But 
$A \geqslant 0$, so $a_{12}^{2} \leqslant a_{11} a_{22}$. Therefore, $a_{11}>0$ and $\mathrm{a}_{22}>0$, and $k^{2}\left(1+a_{11}^{-1}\right)\left(1+a_{22}^{-1}\right)$ $\leqslant 1$. This inequality implies

$$
k^{2}\left(1+a_{11}^{-1}\right)<1 \quad \text { and } \quad k^{2}\left(1+a_{22}^{-1}\right)<1,
$$

whence $a_{11}>1$ and $a_{22}>1$.

In the general case we have $\operatorname{tr} B=k(n+\operatorname{tr} A)>0$, so the largest eigenvalue of $B$ must be positive, while all other eigenvalues are negative or zero. Hence, the quadratic form, $Q$ say, associated with the matrix $B$ is negative or at most equal to zero on a subspace $\Sigma$ of $\mathbf{R}^{n}$ of dimension $n-1$. Now, consider any of the matrices

$$
B_{i j}=\left(\begin{array}{ll}
b_{i i} & b_{i j} \\
b_{i j} & b_{j j}
\end{array}\right), \quad i, j=1, \ldots, n .
$$

If both eigenvalues of $B_{i j}$ were positive, the quadratic form $Q$ would be positive on a two-dimensional subspace of $\mathbf{R}^{n}$. This subspace would certainly have a nontrivial intersection with the subspace $\Sigma$ introduced above, where $Q$ is negative or at most equal to zero. This contradiction leads us therefore to conclude that $\operatorname{det} B_{i j} \leqslant 0$, i.e., $k^{2}\left(1+a_{i i}\right)\left(1+a_{j j}\right) \leqslant a_{i j}^{2}$. The proof is now completed as before.

The following lemma gives the ordering result for the quadratic term in the Riccati equation (2.1).

LEMMA 3. Let $F$ be a given function defined on $(0, \infty)$, whose values are selfadjoint matrices of order $n$ with entries in $L_{\text {loc }}^{1}([0, \infty))$. Let $T$ be chosen such that $T>16 n^{2}$. Suppose the matrix-valued function $R$ satisfies the Riccati equation (2.1) on $[0, T]$. If, for every $t \in[0, T]$, at least $n-1$ eigenvalues of $F(t)$ are $\geqslant 1$, then

$$
\int_{0}^{T} R^{2}(s) d s \geqslant I
$$

Proof. The matrix $R(t)$ is symmetric for all $t \in[0, T]$. Without loss of generality we may assume that the matrix $\int_{0}^{T} R^{2}(s) d s$ is diagonal. We use the following notation:

$$
R(t)=\left(r_{i j}(t)\right), \quad P(t)=\int_{0}^{t} R^{2}(s) d s=\left(\rho_{i j}(t)\right) .
$$

We cover the interval $[0, T]$ by sets $S_{i}, i=0, \ldots, n$, as follows:

$$
\begin{aligned}
& S_{i}=\left\{t \in[0, T]: \sum_{j=1}^{n}\left|r_{i j}(t)\right| \geqslant \frac{1}{4}\left(1+\rho_{i i}(t)\right)\right\}, \quad i=1, \ldots, n, \\
& S_{0}=[0, T] \backslash \bigcup_{i=1}^{n} S_{i} .
\end{aligned}
$$

On $S_{i}(i=1, \ldots, n)$ we have

$$
\rho_{i i}^{\prime}=\sum_{j=1}^{n}\left|r_{i j}\right|^{2} \geqslant \frac{1}{n}\left(\sum_{j=1}^{n}\left|r_{i j}\right|\right)^{2} \geqslant \frac{1}{16 n}\left(1+\rho_{i i}\right)^{2},
$$

so $\rho_{i i}$ is (strictly) increasing, $\left(1+\rho_{i i}\right)^{-1}$ is decreasing, and $-(d / d t)\left(1+\rho_{i i}\right)^{-1} \geqslant$ $1 / 16 n$. Upon integration over $S_{i}$ we obtain the estimate

$$
\text { mes } S_{i} \leqslant 16 n, \quad i=1, \ldots, n \text {. }
$$

Because $T>16 n^{2}$, we conclude that $S_{0}$ is nonempty. 
For any $t$ we have $R(t) \leqslant \tilde{R}(t)$, where $\tilde{R}(t)$ is the diagonal matrix with entries $\sum_{j=1}^{n}\left|r_{i j}(t)\right|$. (Note that $\tilde{R}(t)-R(t)$ is a symmetric diagonally dominant matrix with nonnegative diagonal entries; cf. [4, §7.2].) In particular, if $t \in S_{0}$, then $\sum_{j=1}^{n}\left|r_{i j}\right|$ $<\frac{1}{4}\left(1+\rho_{i i}\right)$ for each $i$, so $R(t) \leqslant \frac{1}{4}(I+\tilde{P}(t))$, where $\tilde{P}(t)$ is the diagonal matrix with entries $\rho_{i i}(t)$. Thus, $I-F(t)=I+P(t)-R(t) \geqslant I+P(t)-\frac{1}{4}(I+\tilde{P}(t))$. Since at least $n-1$ eigenvalues of $I-F(t)$ are $\leqslant 0$, the same is true for the matrix $I+P(t)-\frac{1}{4}(I+\tilde{P}(t))$. (Here we have used Lemma 1.) On the other hand,

$$
\operatorname{tr}\left(I+P(t)-\frac{3}{4}(I+\tilde{P}(t))\right)=\frac{3}{4}(n+\operatorname{tr} P(t))>0,
$$

so the matrix $I+P(t)-\frac{1}{4}(I+\tilde{P}(t))$ has at least one positive eigenvalue. We conclude that this matrix has precisely one positive eigenvalue and $n-1$ eigenvalues which are negative or zero.

Applying Lemma 2 to the matrices $A=P(t)$ and $B=I+P(t)-\frac{1}{4}(I+\tilde{P}(t))$ with $k=\frac{3}{4}$, we see that $\rho_{i i}(t)>1$ for $i=1, \ldots, n$, whenever $t \in S_{0}$. As each $\rho_{i i}$ is a nondecreasing function of $t$, the same inequalities must hold at $t=T$. Because $P(T)$ is diagonal, (2.2) follows.

3. Main result. The ordering relation given in Lemma 3 enables us to compare the quadratic term in (1.4) with multiples of the identity matrix and thus to show that (1.4) does not have a solution on $[a, \infty)$ if $(1.5)$ holds.

THEOREM. If (1.5) holds, then (1.1) is oscillatory at infinity.

Proof. The proof is by contradiction, where we assume that the Riccati equation (1.4) has a selfadjoint matrix-valued solution $R$ on $[a, \infty)$.

If (1.5) holds, then $\lambda_{i}\left\{R(a)+Q_{1}(t)\right\} \rightarrow \infty$ as $t \rightarrow \infty$ for $i=1, \ldots, n-1$, so for $t$ sufficiently large, $t \geqslant t_{0}$ say, we have

$$
\lambda_{i}\left\{R(a)+Q_{1}(t)\right\} \geqslant 1, \quad i=1, \ldots, n-1, t \geqslant t_{0} .
$$

Let $t_{0}$ be kept fixed. For $t \geqslant t_{0}$ we have

$$
R(t)=F_{0}(t)+\int_{t_{0}}^{t} R^{2}(s) d s, \quad t \geqslant t_{0},
$$

where

$$
F_{0}(t)=R(a)+Q_{1}(t)+\int_{a}^{t_{0}} R^{2}(s) d s, \quad t \geqslant t_{0} .
$$

The inequalities (3.1) and the nonnegativity of the integral term imply

$$
\lambda_{i}\left\{F_{0}(t)\right\} \geqslant 1, \quad i=1, \ldots, n-1, t \geqslant t_{0} .
$$

The change of variables $\bar{R}(t)=R\left(t_{0}+t\right)$ reduces (3.2) to

$$
\bar{R}(t)=\bar{F}(t)+\int_{0}^{t} \bar{R}^{2}(s) d s, \quad t \geqslant 0,
$$

where $\bar{F}(t)=F_{0}\left(t_{0}+t\right)$. It follows from (3.4) that $n-1$ eigenvalues of $\bar{F}(t)$ are $\geqslant 1$, so according to Lemma 2 there exists a $\tau>0$ such that $\int_{0}^{\tau} \bar{R}^{2}(s) d s \geqslant I$. Let $t_{1}=t_{0}+\tau$ thus determined be kept fixed. Then

$$
\int_{t_{0}}^{t} R^{2}(s) d s \geqslant I, \quad t \geqslant t_{1}
$$


Proceeding to the next step, we define

$$
F_{1}(t)=F_{0}(t)+\int_{t_{0}}^{t_{1}} R^{2}(s) d s, \quad t \geqslant t_{1} .
$$

Because of (3.4) and (3.6) we have

$$
\lambda_{i}\left\{F_{1}(t)\right\} \geqslant 2, \quad i=1, \ldots, n-1 ; t \geqslant t_{1} .
$$

Furthurmore, from (3.2) it follows that

$$
R(t)=F_{1}(t)+\int_{t_{1}}^{t} R^{2}(s) d s, \quad t \geqslant t_{1} .
$$

The change of variables $\bar{R}(t)=\frac{1}{2} R\left(t_{1}+\frac{1}{2} t\right)$ transforms this equation into (3.5), where $\bar{F}(t)=\frac{1}{2} F_{1}\left(t_{1}+\frac{1}{2} t\right)$. Thus, $\lambda_{i}\{\bar{F}(t)\} \geqslant 1$ for $i=1, \ldots, n-1$ and Lemma 2 applies. Adjusting the value of $\tau$ if necessary, we conclude that $\int_{0}^{t} \bar{R}^{2}(s) d s \geqslant I$ for $t \geqslant \tau$, i.e.,

$$
\int_{t_{1}}^{t} R^{2}(s) d s \geqslant I, \quad t \geqslant t_{2}
$$

where $t_{2}=t_{1}+\frac{1}{2} \tau=t_{0}+\frac{3}{2} \tau$.

Continuing this process we find, after $n$ steps,

$$
\int_{t_{n-1}}^{t} R^{2}(s) d s \geqslant I, \quad t \geqslant t_{n}
$$

where $t_{n}=t_{n-1}+\tau / 2^{n-1}=t_{0}+2\left(1-2^{-n}\right) \tau$. Thus, adding the contributions of $\left[a, t_{0}\right],\left[t_{0}, t_{1}\right], \ldots,\left[t_{n-1}, t_{n}\right]$, we obtain the estimate

$$
\int_{a}^{t} R^{2}(s) d s \geqslant\left(2^{n}-1\right) I, \quad t \geqslant t_{n} .
$$

But $t_{n}$ tends to the finite limit $t_{0}+2 \tau$ as $n \rightarrow \infty$, so there exists a finite number $T$ such that $R(t)$ blows up as $t \uparrow T$. This conclusion contradicts the assumption that (1.4) has a solution on $[a, \infty)$.

A closer examination of the proof of Lemma 3 shows that all that is needed for the lemma to hold is that enough (namely, $n-1)$ eigenvalues of the matrix $I+P(t)-\frac{1}{4}(I+\tilde{P}(t))$ are less than or equal to zero. The condition that $n-1$ eigenvalues of $F(t)$ are greater than or equal to one is sufficient, but by no means necessary for this to be true. Consequently, the condition (1.5) is only sufficient for (1.1) to be oscillatory at infinity.

Also, the condition (1.5) need not hold for all sufficiently large $t \mathrm{n}$. If the eigenvalues grow beyond any bound on a sufficiently large set of $t$-values, the conclusion remains valid. We refer the reader to our earlier publication [2], where this idea is pursued in some detail.

\section{REFERENCES}

1. D. Hinton and R. T. Lewis, Oscillation theory for generalized second-order differential equations, Rocky Mountain J. Math. 10 (1980), 751-766.

2. M. K. Kwong and H. G. Kaper, Oscillation of two-dimensional linear second-order differential systems, J. Differential Equations 56 (1985).

3. M. K. Kwong, H. G. Kaper, K. Akiyama and A. B. Mingarelli, Oscillation of linear second-order differential systems, Proc. Amer. Math. Soc. 91 (1984), 85-91. 
4. P. Lancaster, Theory of matrices, Academic Press, New York, 1969.

5. A. B. Mingarelli, On a conjecture for oscillation of second-order ordinary differential systems, Proc. Amer. Math. Soc. 82 (1981), 593-598.

6. An oscillation criterion for second-order selfadjoint differential systems, C. R. Math. Rep. Acad. Sci. Canada 2 (1980), 287-290.

7. W. T. Reid, Sturmian theory for ordinary differential equations, Springer-Verlag, New York, 1980.

Mathematics and Computer Science Division, Argonne National laboratory, Argonne, ILLINOIS 60439 (Current address of H. G. Kaper)

Current address (F. V. Atkinson): Department of Mathematics, University of Toronto, Toronto M5S 1A1, Ontario, Canada

Current address (M. K. Kwong): Department of Mathematical Sciences, Northern Illinois University, DeKalb, Illinois 60115 